\title{
Quality Control of Dual Energy X-Ray Absorptiometry
}

\author{
M. A. Reza1 ${ }^{*}$, A. K. Paul', H. R. Khan'1, M. R. Rahman'1 \\ ${ }^{1}$ Physics Discipline, Khulna University, Khulna, Bangladesh \\ ${ }^{2}$ Institute of Nuclear Medicine \& Allied Sciences, Khulna, Bangladesh \\ Email: *reza101710@gmail.com
}

How to cite this paper: Reza, M.A., Paul, A.K., Khan, H.R. and Rahman, M.R. (2018) Quality Control of Dual Energy X-Ray Absorptiometry. International Journal of Medical Physics, Clinical Engineering and Radiation Oncology, 7, 323-329.

https://doi.org/10.4236/ijmpcero.2018.73026

Received: January 26, 2018

Accepted: July 30, 2018

Published: August 2, 2018

Copyright () 2018 by authors and Scientific Research Publishing Inc. This work is licensed under the Creative Commons Attribution International License (CC BY 4.0).

http://creativecommons.org/licenses/by/4.0/

\begin{abstract}
Dual energy X-ray absorptiometry (DXA) is a dominant technique for the measurement of bone mineral density (BMD). Quality control (QC) of DXA is very important for the accuracy of results and correct interpretation made by the physician. We have performed the quality control procedures of Lunar DPX Pro bone densitometer according to the manufacturer's recommendations and current clinical guidelines at the Institute of Nuclear Medicine \& Allied Sciences (INMAS), Khulna. The objective of this study is to maximize the performance of the technologist as well as the reliability of the equipment (Linearity, X-ray tube output, Half value layer, Kerma-area product, Radiation field size, Fan angle, Spatial resolution, Room safety). The study result shows that the mean BMD reading is $1.004 \mathrm{~g} / \mathrm{cm}^{2}$ with a standard deviation of 0.0035 and co-efficient of variation $0.34 \%$. It also shows that the precision of the technologist is good and there is no malfunctioning in the DXA bone densitometer.
\end{abstract}

\section{Keywords}

Nuclear Medicine, DXA, Bone Mineral Density, Quality Control, Bone Densitometer

\section{Introduction}

Osteoporosis is a prolonged condition of multifactorial etiology involving systemic skeleton characterized by decreased bone mass and deterioration of bony microarchitecture. The result is fragile bones and an increased risk of fractures, even after subtle trauma [1]. According to the World Health Organization (WHO) criteria, osteoporosis is defined as a BMD value less than or equal to 2.5 standard deviations below the young normal mean reference population [2]. 
Currently, it is estimated that over 200 million people worldwide suffer from this disease, and at least $40 \%$ of women and about $20 \%$ of men will sustain one or more fragility fractures in their lifetime [3]. In 1987, estimation of BMD using dual energy X-ray absorptiometry (DXA) was introduced for routine clinical use and in 1994, World Health Organization declared DXA as the gold standard for BMD estimation in its technical report [4]. The proliferation of devices to measure BMD raises concern regarding the quality of the studies. When performing DXA measurements, it is important to implement a QC program to guarantee that any observed density changes are real and not due to machine or to operator variability. Incorrect BMD acquisition or reporting may generate unnecessary medical expenses and lead to inappropriate clinical and therapeutic decisions that could have adverse effects on patients' health [5]. Mistakes in BMD valuation can result from lack of adherence to manufacturers' recommendations for device maintenance and quality control, inappropriate acquisition and analysis of data. In 2006, Lewiecki et al. [6] reported about $71 \%$ of clinicians and $45 \%$ of technologist see incorrect densitometric interpretation and acquisition at least once a month. Hence, a comprehensive quality control (QC) system is essential step to help the management of patients. Current research has focused on the current practice of quality control (QC) procedures in bone densitometry. In order to study the QC of the 3rd generation-pencil beam (PB) DXA equipment in the bone densitometry unit of INMAS, Khulna, the equipment performance \& technologist's expertise were assessed to make sure that the equipment performance meets the manufacturer's specifications and technologist's precision does not exceed the International Society for Clinical Densitometry (ISCD) issued standards. The rest of the article is structured as follows: first, the equipments used in this study are presented briefly. This is followed by a description of the research methods and procedures used in the study. The results and discussions of our study are then summarized and discussed. The paper concludes with a conclusion of the study's research contributions.

\section{Equipment}

Equipment packages includes dedicated phantom for calibration and QC procedures, the positioning device for the anteroposterior lumbar spine scan acquisition; the hip positioning device for the femur scan acquisition etc. Which are shown in Figure 1.

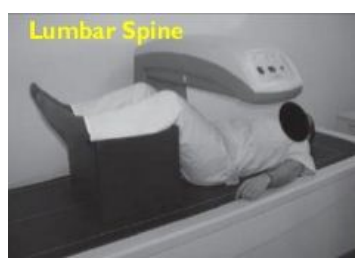

(a)

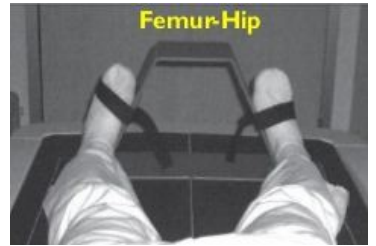

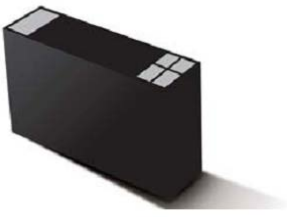

(b)

Figure 1. (a) Patient positioning; (b) Lunar aluminum spine phantom. 
Radiation dosimeter, Aluminum filters and X-ray film cassette were used in acceptance testing and routine quality control (QC) of DXA machine. These essential elements are shown in Figure 2.

\section{Methodology}

This study was done under a designed protocol developed by International Radiation Protection Association (IRPA), Bulgaria in compliance with international standards (ISCD, IOF) at the BMD unit of INMAS, Khulna during the period of June 2014 to November 2014. A detailed description of the protocol is provided by Slavchev, A. et al. (2008) [7]. The QC of DXA involves the integration of several processes: functional inspection of the equipment, radiation safety measure and in-vitro precision. The functional inspection of the equipment consists of a series of standard periodic tests which includes X-ray tube output, HVL, field size, surface air kerma, Kerma area product (KAP), Spatial resolution, fan angle etc. The consistency of the X-ray tube output was measured by placing the survey meter onto the patient table and performing ten scans in the lumbar spine mode. In addition HVL was measured with survey meter positioned $10 \mathrm{~cm}$ above the patient table and by placing $\mathrm{Al}$ filters of different thickness underneath until the dose falls to below $50 \%$ of the un-attenuated value. We calculated the radiation field size by a film cassette. The schematic diagram of the measurement is shown in Figure 3. After calculating the field size we measured the surface air kerma on the entrance surface of the phantom, positioned above the survey meter lying on the patient table for different scan modes. Then Kerma

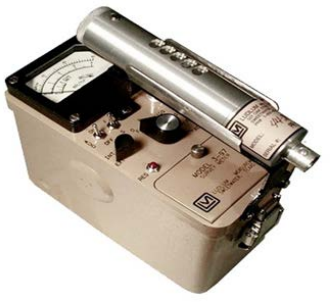

(a)

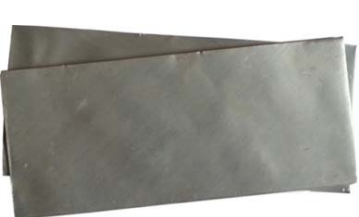

(b)

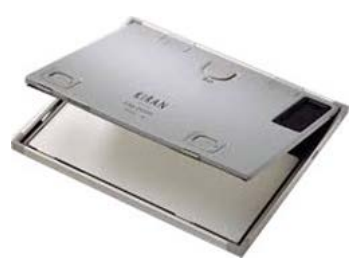

(c)

Figure 2. (a) Radiation dosimeter, (b) Aluminum filters and (c) X-ray film cassette.

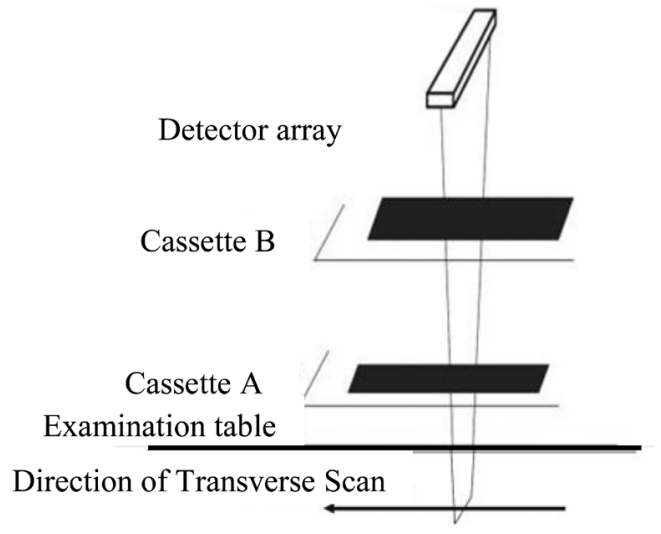

Figure 3. Schematic drawing of fan angle measurement process. 
area product (KAP) was calculated from the measured values and the corresponding area. The data is shown in Table 1. Spatial resolution of the machine was tested by mounting a test tool over the X-ray film cassette lying on the patient table. An acquisition is performed using lumbar spine mode such that the radiation beam covers exactly the inner pattern of the test tool. For the estimation of fan angle an acquisition is performed using lumbar spine mode with an $\mathrm{X}$-ray film cassette " $\mathrm{A}$ " placed on the patient table and another cassette " $\mathrm{B}$ " at 20 $\mathrm{cm}$ above cassette " $\mathrm{A}$ ". The width value of exposed areas was recorded.

To evaluate the accuracy of the DXA, a daily calibration measurement, according to the manufacturer's instructions, is performed using the standard phantom supplied with the system. To find machine drifts occurring over time visual inspection of CUSUM chart was used which is shown in Figure 4. In order to utilize the CUSUM chart, a baseline spine phantom value was established by scanning the phantom once on each of 25 consecutive days. For all subsequent scans, the difference between the average value and the subsequent value was calculated. The differences are progressively summed and plotted on the CUSUM chart. To investigate the radiation safety scattered radiation was measured at different distances from the phantom placed on the patient table with the survey meter. Short-term precision was examined using 15 patients; three tests per patient were done. The data collected in precision test is shown in Table 2. In order to assess the measurement error accurately the patient were repositioned during each test. And then precision error is calculated.

\section{Results and Discussion}

We found that the X-ray tube output is consistent to $4.78 \%$ which indicates that the machines exhibit a good reproducibility of the generated output. The measured value of first $\mathrm{HVL}$ was $4.7 \mathrm{~mm} \mathrm{Al}$ (with tube voltage $76 \mathrm{KeV}$ and tube

Table 1. Evaluation of acceptance testing parameters for DXA machine.

\begin{tabular}{|c|c|c|c|c|c|c|}
\hline \multicolumn{4}{|c|}{$\mathrm{X}$-ray tube output at $0.375 \mathrm{~mA}$} & \multicolumn{2}{|c|}{ Half value layer at $76 \mathrm{Kv}, 0.375 \mathrm{~mA}$} & \multirow{2}{*}{$\begin{array}{c}\text { Scatter } \\
\text { Exposure on } \\
\text { Work station } \\
\text { (in } \mu S v \text { ) }\end{array}$} \\
\hline $\begin{array}{l}\text { Scan } \\
\text { No. }\end{array}$ & Scan time (s) & $\mathrm{mA} \times \mathrm{s}$ & $\begin{array}{l}\text { Radiation } \\
\text { output }\end{array}$ & $\begin{array}{l}\text { Thickness of } \\
\mathrm{Al} \text { (in } \mathrm{mm} \text { ) }\end{array}$ & $\begin{array}{l}\text { Dose received } \\
\quad \text { (in } \mu S v \text { ) }\end{array}$ & \\
\hline Scan 1 & 164 & 61.500 & 10.00 & Without filter & 10.15 & 0.40 \\
\hline Scan 2 & 159 & 59.625 & 09.80 & 0.6 & 09.80 & 0.40 \\
\hline Scan 3 & 163 & 61.125 & 09.80 & 1.2 & 09.80 & 0.35 \\
\hline Scan 4 & 165 & 61.875 & 09.60 & 1.8 & 09.00 & 0.40 \\
\hline Scan 5 & 161 & 60.375 & 10.20 & 2.4 & 08.00 & 0.40 \\
\hline Scan 6 & 163 & 61.125 & 11.00 & 3.0 & 07.00 & 0.40 \\
\hline Scan 7 & 164 & 61.500 & 09.20 & 3.6 & 06.00 & 0.35 \\
\hline Scan 8 & 163 & 61.125 & 09.80 & 4.2 & 06.00 & 0.35 \\
\hline Scan 9 & 165 & 61.875 & 10.30 & 4.8 & 05.00 & 0.40 \\
\hline Scan 10 & 158 & 59.250 & 10.00 & 5.4 & 05.00 & 0.40 \\
\hline
\end{tabular}




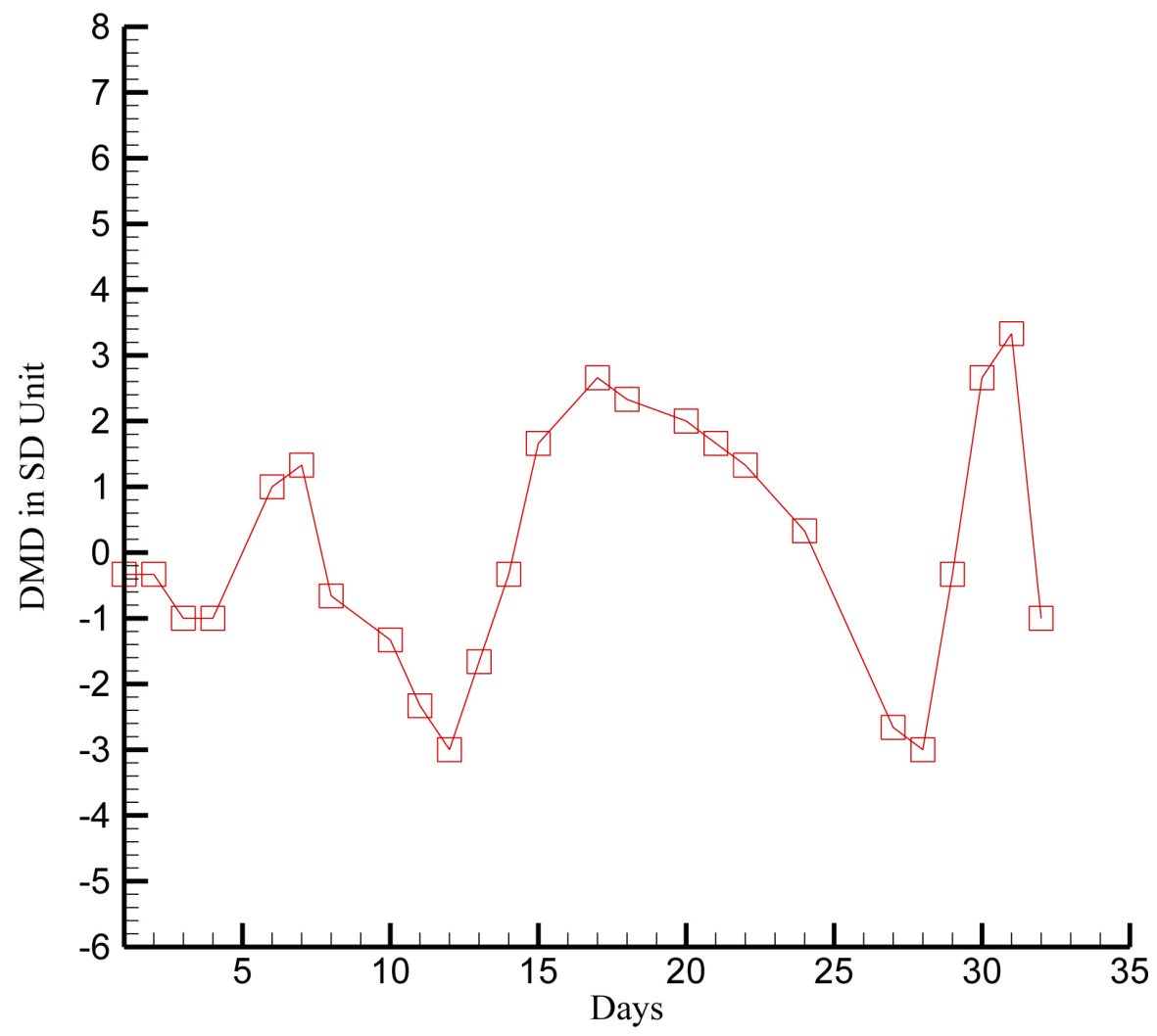

Figure 4. A CUSUM chart. The measured values plotted on the CUSUM chart are scattered in a horizontal pattern around 0 ( 0 is equal to the average phantom value).

Table 2. The measured lumbar spine values for 15 patients in a short-term precision study.

\begin{tabular}{cccccccccc}
\hline Subject & Scan 1 & Scan 2 & Scan 3 & Mean & Subject & Scan 1 & Scan 2 & Scan 3 & Mean \\
\hline $\mathbf{1}$ & 1.153 & 1.151 & 1.158 & 1.154 & $\mathbf{0 9}$ & 0.881 & 0.92 & 0.879 & 0.893 \\
$\mathbf{2}$ & 1.131 & 1.114 & 1.115 & 1.120 & $\mathbf{1 0}$ & 0.907 & 0.909 & 0.921 & 0.912 \\
$\mathbf{3}$ & 0.903 & 0.893 & 0.907 & 0.901 & 11 & 1.185 & 1.181 & 1.181 & 1.182 \\
$\mathbf{4}$ & 1.099 & 1.098 & 1.105 & 1.101 & $\mathbf{1 2}$ & 1.108 & 1.099 & 1.101 & 1.103 \\
$\mathbf{5}$ & 1.117 & 1.117 & 1.173 & 1.136 & 13 & 1.311 & 1.307 & 1.307 & 1.308 \\
$\mathbf{6}$ & 1.110 & 1.119 & 1.081 & 1.103 & 14 & 1.047 & 1.043 & 1.047 & 1.046 \\
$\mathbf{7}$ & 1.164 & 1.175 & 1.177 & 1.172 & 15 & 1.017 & 0.997 & 1.002 & 1.005 \\
$\mathbf{8}$ & 1.173 & 1.167 & 1.168 & 1.169 & & & & & \\
\hline
\end{tabular}

current $0.375 \mathrm{~mA}$ ). Lunar (Pencil Beam) System with $76 \mathrm{kVp}$ k-edge filter has half value layer of $3.6-4.5 \mathrm{~mm} \mathrm{Al}$ [8]. The HVL thickness has excided the manufacturer specification which causes less beam hardening. In spine scan mode, the scan area or the area of the irradiated field was $17.8 \mathrm{~cm} \times 16.5 \mathrm{~cm}$ with a maximum line scan of 167 lines. The measured Kerma Area Product (KAP) is $2.94 \mathrm{~cm}^{2}$. This KAP can vary over an order of magnitude from 2 to $36 \mathrm{~cm}^{2}$ in the range of systems studied [9].

For this modality the spatial resolution was found $0.5-1.0 \mathrm{l} / \mathrm{mm}$ in the 
transverse direction. The measured fan beam angle in the longitudinal direction is 0.1725 degree representing no significant fanning of the beam. Scatter measurement found that the scatter dose on operators' desk positioned at $1 \mathrm{~m}$ away from the central axis of the patient table was $0.34 \mu S v$ which indicates the operator dose is in the lower range of acceptable occupational exposures [4] [5]. The visual inspection of CUSUM chart "Figure 2" shows the phantom BMD values are randomized rather than gradually rising or falling representing the proper functioning of the machine.

The precision error and LSC estimated by using 15 subjects scanned three times was $0.014 \%$ and $3.88 \%$ respectively which is within the ISCD recommended limits reflecting expertise of the technologist.

\section{Conclusion}

The present study has investigated mechanical acceptance testing, occupational radiation exposure and technologist precision. Among the aforementioned QC tests, all testing parameters except HVL value of the filter are in good agreement with the manufacturer supplied acceptance limit. The technologist precision is also good. Any apparatus that exposes patients to ionising radiation should be subject to regular performance checks. This study on QC of DXA will assist with the on-going development of QC testing guidelines, determination of required testing frequencies, the establishment of tolerances, and criteria of acceptability for DXA X-Ray equipment.

\section{Acknowledgements}

We would like to gratefully acknowledge to Dr. Ashoke Kumar Paul, Director and Chief Medical Officer, Institute of Nuclear Medicine \& Allied Sciences (INMAS), Khulna, for his cordial cooperation, providing required facilities needed to complete this research successfully.

\section{Conflicts of Interest}

The authors declare no conflicts of interest regarding the publication of this paper.

\section{References}

[1] Zaman, M.U., et al. (2009) Dual X-Ray Absorptiometry (DXA): Basic Techniques and Clinical Facts. Pakistan Journal of Radiology, 19, 56-59.

[2] Guglielmi, G., et al. (2014) Quality Assurance in Bone Densitometry. Current Cardiology Reports, 2, 33. https://doi.org/10.1007/s40134-013-0033-9

[3] World Health Organization (2007) Assessment of Osteoporosis at the Primary Health Care Level. Summary Report of a WHO Scientific Group.

[4] World Health Organization (1994) Assessment of Fracture Risk and Its Application to Screening for Postmenopausal Osteoporosis: Technical Report Series 843. WHO, Geneva.

[5] Lewiecki, E.M. and Lane, N.E. (2008) Common Mistakes in the Clinical Use of Bone 
Mineral Density Testing. Nature Clinical Practice, Rheumatology, 4, 667-674. https://doi.org/10.1038/ncprheum0928

[6] Lewiecki, E.M., Binkley, N. and Petak, S.M. (2006) DXA Quality Matters. Journal of Clinical Densitometry, 9, 388-392. https://doi.org/10.1016/j.jocd.2006.07.002

[7] Slavchev, A., et al. (2008) National Protocol for Quality Assurance in DXA-Bone Densitometry. Polish Journal of Medical Physics and Engineering, 14, 207-215. https://doi.org/10.2478/v10013-008-0017-x

[8] Larkin, A., et al. (2008) Commissioning and Quality Assurance of Dual Energy X-Ray Absorptiometry (DEXA) System. Radiation Protection Dosimetry. (In Press)

[9] Sheahan, N.F., et al. (2005) Commissioning and Quality Assurance Protocols for Dual Energy X-Ray Absorptiometry (DEXA) Systems. Radiation Protection Dosimetry, 117, 288-290. https://doi.org/10.1093/rpd/nci741 